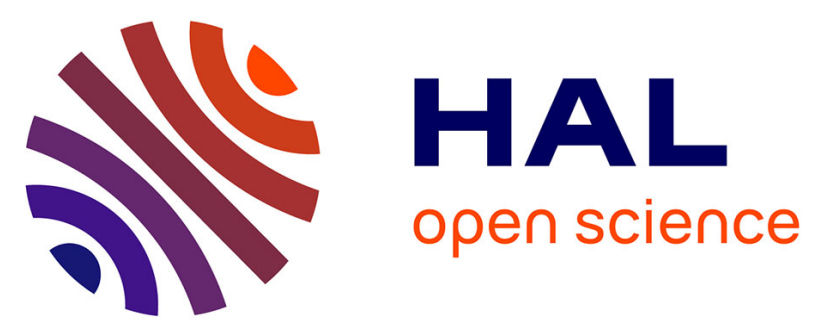

\title{
Study and modeling of fluctuating dissolved oxygen concentration impact on Corynebacterium glutamicum growth in a scale-down bioreactor
}

Abdoul Karim Kaboré, Stéphane Delaunay, Fabrice Blanchard, Emmanuel Guedon, Michel Fick, Eric Olmos

\section{To cite this version:}

Abdoul Karim Kaboré, Stéphane Delaunay, Fabrice Blanchard, Emmanuel Guedon, Michel Fick, et al.. Study and modeling of fluctuating dissolved oxygen concentration impact on Corynebacterium glutamicum growth in a scale-down bioreactor. Process Biochemistry, 2018, 10.1016/j.procbio.2018.10.016 . hal-01924081

\section{HAL Id: hal-01924081 \\ https://hal.univ-lorraine.fr/hal-01924081}

Submitted on 21 Oct 2021

HAL is a multi-disciplinary open access archive for the deposit and dissemination of scientific research documents, whether they are published or not. The documents may come from teaching and research institutions in France or abroad, or from public or private research centers.
L'archive ouverte pluridisciplinaire HAL, est destinée au dépôt et à la diffusion de documents scientifiques de niveau recherche, publiés ou non, émanant des établissements d'enseignement et de recherche français ou étrangers, des laboratoires publics ou privés.

\section{(c)(1) $\$$}

Distributed under a Creative Commons Attribution - NonCommerciall 4.0 International 


\section{Study and modeling of fluctuating dissolved oxygen concentration impact on Corynebacterium glutamicum growth in a scale-down bioreactor.}

Abdoul Karim Kaborée,b, Stéphane Delaunay ${ }^{\mathrm{a}, \mathrm{b}}$, Fabrice Blanchard ${ }^{\mathrm{a}, \mathrm{b}}$, Emmanuel Guedon $^{\mathrm{a}, \mathrm{b}}$, Michel Fick ${ }^{\mathrm{a}, \mathrm{b}}$, Eric Olmos ${ }^{\mathrm{a}, \mathrm{b}, *}$

${ }^{a}$ CNRS, Laboratoire Réactions et Génie des Procédés, UMR 7274, 2 avenue de la forêt de Haye, TSA 40602, Vandouvre-lès-Nancy, F-54518, France

${ }^{b}$ Université de Lorraine, LRGP, UMR 7274, 2 avenue de la forêt de Haye, TSA 40602, Vandœuvre-lès-Nancy, F-54518, France

${ }^{*}$ Corresponding author : eric.olmos@univ-lorraine.fr

Preprint submitted to Process Biochemistry

October 10, 2018 


\begin{abstract}
In this study, the impact of dissolved oxygen concentrations oscillations on Corynebacterium glutamicum $2262 \Delta \mathrm{ldhA}$ growth was studied experimentally and modeled. Aiming at this, a dedicated two-compartment scale down set-up composed of two interconnected aerobic/anaerobic stirred tank bioreactors was used. The mean residence time of bacteria in each compartment was modified by adapting circulation rates and culture volumes in each bioreactor and the resulting temporal ratio of aeration was calculated. The five growth kinetics were then modeled using an original kinetic model coupling Monod growth modeling and the Residence Time Distributions. Our study showed that the microbial growth rate and macroscopic yields were clearly linked to the temporal ratio of aeration, allowing the definition of simple but robust law for process scale-up purpose. It was also revealed that the model proposed precisely agreed with the experimental growth data, whatever the fractions of aeration time imposed experimentally.
\end{abstract}

\title{
Keywords
}

Scale-down, kinetic model, Corynebacterium glutamicum 
1

2

3

4

5

\section{Introduction}

Whereas the first step of process development generally consists in the research of value-added products production at the laboratory scale by selecting productive microbial strains for instance, scale-up of bioreactor is the second paramount step to allow the large-scale synthesis of the targeted molecule. However, using a constant power dissipation per unit of volume as criterion for bioreactor scale-up entails an increase of mixing times at larger scales [1], that potentially promotes concentration heterogeneities. Therefore, prediction, at lab-scale, of possible impact of concentration gradients on microbial performance is of great interest before scale-up and was the subject of major publications these last years $[2,3,4,5,6]$. The concentration gradients observed may concern dissolved oxygen concentrations $[7,8,9], \mathrm{pH}[10]$ or nutrients such as carbon source [11]. Among the previous parameters identified, dissolved oxygen is one of the most critical, due to heterogeneity of the oxygen injection, to spatial variation of hydrostatic pressure and thus of oxygen transfer capacities and to high oxygen consumption rates when high biomass concentrations are encountered [12]. To investigate the physiological effects of chronic exposure of microorganisms to the heterogeneous distribution of micro-environmental conditions, scale-down models were then introduced as rational and practical approaches to simulate the dynamic micro-environment (also called 'lifelines') experienced by the cells at large scale by using small-scale set-ups. In most studies, fluctuating environments are controlled by combining two or three small-scale bioreactors [2].

In this study, the behaviour of Corynebacterium glutamicum subjected to fluctuations of dissolved oxygen concentrations, is studied using a scale-down approach. 
Corynebacterium glutamicum is a gram-positive bacterium isolated in 1950s, used for the production of millions tons of amino acid per year including, glutamate, lysine, valine and alanine $[13,14]$. Currently, the interest for $C$. glutamicum as an efficient organic acids producer under deprived oxygen conditions is increasing $[15,16]$. To meet the demand of bio-based products, optimized large-scale bioreactors are required, thus leading to the occurrence of possible spatial concentration gradients. Until now, only few scale-down approaches have been carried out with C. glutamicum. Schilling et al. [17] investigated the performance of L-lysine production by $C$. glutamicum under substrate gradients while glucose and oxygen oscillations impact on C. glutamicum metabolism have been analyzed recently in a Continuous Stirred Tank (CST) bioreactor connected to Plug Flow (PF) bioreactor [18, 19, 20] or connected to another CST [21]. In all these works, the robustness of C. glutamicum towards fluctuations of carbon source or oxygen concentrations was noticed. However, although no modification at transcriptomic, proteomic and metabolomics levels was noticed [18], oscillations induced a modification in the carbon fluxes resulting in an accumulation of side-products such as lactate $[19,20,21]$. These negative effects were increased when C. glutamicum was cultivated in a complex medium. In this culture conditions, in a three-compartment scale-down reactor, a significative decrease in the product yield, a stop of growth and an accumulation of lactate and acetate were observed [20]. Otherwise, Buchholz et al. [22] focused on C. glutamicum metabolic and transcriptional response when exposed to carbon dioxide gradients in a three stirred tank compartment set-up. They reported that the dissolved $\left[\mathrm{CO}_{2}\right]$ gradients expected at industry-relevant residence times, of about $3.6 \mathrm{~min}$, did not 
hamper the process performances in comparison to lab-scale reference. However, transcriptional analysis have revealed up to 66 differentially expressed genes already after a $3.6 \mathrm{~min}$ dissolved $\left[\mathrm{CO}_{2}\right]$ gradient exposure ; the genes expression was also related to gradient intensity and residence time of the cells. More recently, advanced CFD simulations using Euler-Lagrangian approaches were performed to determine the substrate concentrations encountered by cells in industrial scale bioreactors by Haringa et al. [23, 24, 25]. These approaches allow the design of adapted and representative scale-down set-ups. In our previous work, the variation of DOC imposed between fully-aerobic growth and oxygen-limited production phases was shown to play an important role in the succinic acid production process [26]. Therefore, it seems of great importance to also determine this impact when the bacteria are exposed to chronic gradients, as encountered in large-scale bioreactors. The present work is thus dedicated to the study of $C$. glutamicum response when this microorganism is subjected to DOC oscillations, by using a CSTR-CSTR scale-down set-up. Whereas one CSTR operate in anaerobiosis, the second one operates in fully-aerobiosis. As presented by Limberg et al. [21], CST are characterized by a high back-mixing, offering high level of flexibility for the adjustment of mean residence time and the control of DOC, temperature and $\mathrm{pH}$ in both compartments. However, as the Residence Time Distribution (RTD) of CSTR are strongly spread around the mean residence time, with a standard deviation equal to the mean residence time $\tau$, it is necessary to consider the impact of RTDs on the real DOC fluctuations imposed to the cells. To manage this, our study proposes an approach that takes into account the DOC fluctuations along bacteria lifelines and their impact on microbial growth, in the 
CSTR-CSTR loop. The results of this model are then compared to the experimental measurements.

\section{Material and methods}

\subsection{Bacterial strain, culture media and inoculum preparation}

Batch cultures of a mutant strain of C. glutamicum 2262, deleted for lactate dehydrogenase gene $(\Delta \mathrm{ldh} A)$ were performed in a modified MCGC mineral salt medium in which citrate was replaced by deferoxamine. This mutant strain is built using the minimal genetic modification to redirect the carbon flux towards the succinate production as presented in a previous paper [27]. Final concentrations of nutrients and medium preparation details are provided in a previous study [26]. The mutant strain was constructed via a two-step homologous recombination procedure using the vector pK19mobsacB $\Delta$ ldh 34 . This plasmid was constructed using the suicide vector pK19mobsacB as previously described [28]. The culture medium was supplemented with glucose as carbon and energy source at the desired concentration. Inoculum culture is obtained from glycerol stocks used in different preculture steps in mineral salts medium and described in the study of Kaboré et al. [26].

\subsection{Experimental set-up and culture conditions}

To impose oscillating dissolved oxygen concentration (DOC) to cultures of $C$. glutamicum $2262 \Delta \mathrm{ldhA}$, a scale-down bioreactor system was used. This system consisted of two interconnected stirred tank bioreactors (Tryton, Pierre Guerin Technologies, France). One bioreactor was maintained at a constant DOC of $50 \%$ of air saturation $\left(\mathrm{Bio}_{\mathrm{O} 2}\right)$ while the second was set at $\mathrm{DOC}=0 \%$ of air saturation $\left(\mathrm{Bio}_{N_{2}}\right)$ 
to mimic transition from aerobic to anaerobic environments (Figure 1). The culture broth continuously looped between both vessels at given recirculation flow rates $Q$ through a low gas permeability tubing (Exacanal, $5 \mathrm{~mm}$ internal diameter, $2.5 \mathrm{~m}$ long and volume $V_{\text {tube }}=50 \mathrm{~mL}$ ) using two peristaltic pumps (Masterflex, Cole-Parmer, Barrington IL, USA). Depending on liquid volume, each bioreactor was equipped with two or three four-bladed Rushton turbines (diameter $D=0.06 \mathrm{~m}$ ). DOC was measured in both bioreactors using sterilizable polarographic probes (Mettler-toledo, ISM technology, Switzerland). In the aerobic $\mathrm{Bio}_{2}$ bioreactor, DOC was maintained constant by sparging air or pure oxygen through a ten-orifice diffuser and by adjusting gas flow rate. Anaerobic conditions were ensured in $\mathrm{Bio}_{N 2}$ bioreactor by sparging pure nitrogen through a porous diffuser. Liquid volumes $V_{1}$ and $V_{2}$ and residence times $\tau_{1}=V_{1} / Q$ and $\tau_{2}=V_{2} / Q$ in aerobic and anaerobic bioreactors respectively were set-up according to Table 1. Agitation rates were set to constant values of $900 \mathrm{rpm}$ and $500 \mathrm{rpm}$ respectively for $\mathrm{Bio}_{2}$ and $\mathrm{Bio}_{N 2}$. $\mathrm{pH}$ was measured using sterilizable glass electrode (Mettler-toledo, ISM technology, InPro3253, Switzerland) and regulated in the two compartments at a set-point of 7.6 by automatic addition of a $10 \mathrm{~N} \mathrm{KOH}$ solution or $2 \mathrm{~N} \mathrm{HCl}$ with a peristaltic pump connected to an on/off $\mathrm{pH}$ controller. The foam formation was controlled by manual addition of drops of pure polypropylene glycol (ppg) in both vessels with respect to final concentration less than $1.2 \mathrm{~mL}$ ppg $\mathrm{L}^{-1}$ of broth. The temperature of cultures was maintained at $33^{\circ} \mathrm{C}$ by automatic cool and hot water circulation in the jacket of the bioreactors. Biomass concentrations, glucose and organic acids concentrations were measured following the protocols detailed in the work of Kaboré et al. [27]. The precision of 
biomass concentration determination was $5 \%$ and the one of glucose and organic acid concentrations was $3 \%$. Three different control cultures were first performed at constant DOC. The first one, $\mathrm{Bio}_{r e f 1}$, consisted of a culture performed using a single aerobic bioreactor and thus without medium circulation ; this experiment was identified as reference for biomass growth maximization. To assess the impact of twocompartment experimental set-up on microbial response, a second reference culture $\mathrm{Bio}_{r e f 2}$ consisting of two identical aerobic CSTRs connected by the continuous recirculation was studied (Table 1). For the third reference culture Bio $_{r e f 3}$, two CSTRs operating in anaerobic conditions were interconnected and were considered as reference cultures of $C$. glutamicum $2262 \Delta l d h A$ regarding organic acids production. In addition to these three reference cultures, six culture conditions imposing fluctuating oxygenation conditions were defined. These experiments could be described by their mean aeration ratios and residence times :

$$
\alpha \simeq \frac{\tau_{1}+V_{\text {tube }} / Q}{\tau_{1}+\tau_{2}+2 V_{\text {tube }} / Q}
$$

Lastly, to quantify the culture performance in terms of growth, uptake and production, maximal specific growth rate $\mu_{\max }$ and maximal yield of biomass on glucose $Y_{\max }^{X / S}$ were determined using method of Kaboré et al. [27].

\section{Results}

\subsection{Control cultures at constant dissolved oxygen concentration}

Kinetics of growth, consumption of glucose, production of organic acids for the anaerobic culture, pH and DOC profiles are shown in Figure 2 for the three reference 
cultures detailed in Table 1. Biomass concentration increased exponentially until exhaustion of glucose with maximal biomass concentrations of 18.2 and $19.8 \mathrm{~g}_{C D W}$ $\mathrm{L}^{-1}$, respectively obtained in one aerobic and two aerobic interconnected bioreactors (Figure 2A 2B). Only minor amounts of organic acids were detected in the aerobic control cultures including pyruvate, acetate with maximal concentrations of $0.4 \mathrm{~g}$ $\mathrm{L}^{-1}$ approximately and succinate with a maximal concentration of $1 \mathrm{~g} \mathrm{~L}^{-1}$. For the anaerobic reference culture $\mathrm{Bio}_{\text {Ref3, }}$, a negligible growth with maximal biomass concentration of $0.7 \mathrm{~g}_{C D W} \mathrm{~L}^{-1}$ was measured after $50 \mathrm{~h}$ of culture (Figure $2 \mathrm{C}$ ). Whereas for the aerobic cultures the initial amount of glucose was completely exhausted, only $29 \mathrm{mM}\left(4.3 \mathrm{~g} \mathrm{~L}^{-1}\right)$ were consumed for the anaerobic culture (Figure 2D). In these anaerobic conditions, the glucose consumed was mainly converted into organic acids. After $50 \mathrm{~h}$ of culture also, the maximal concentrations of pyruvate, acetate and succinate were respectively $4.6 \mathrm{mM}\left(0.4 \mathrm{~g} \mathrm{~L}^{-1}\right), 10.9 \mathrm{mM}\left(0.7 \mathrm{~g} \mathrm{~L}^{-1}\right)$ and $14.6 \mathrm{mM}(1.7$ $\mathrm{g} \mathrm{L}^{-1}$ ) (Figure 2D). These concentrations were similar to the one measured in aerobic conditions but with 30 times less of biomass. The linearity of the organic acids production kinetics seemed to indicate that this production was mainly related to biomass concentration and not to specific growth rate. From the results obtained, it could be concluded that (i) similar results were obtained in the two-compartment and single compartment aerobic bioreactors, (ii) in fully-anaerobic cultures, no growth but organic acids production was observed, (iii) in fully-aerobic culture conditions, exponential growth but no significant organic acids production could be measured. 


\subsection{Modeling of oxygen fluctuations imposed to C. glutamicum}

For the experiments with aeration ratios $0<\alpha<1$ (experiments 1 to 6 in Table 1 ), fluctuations of DOC were imposed by continuously circulating cells from the aerobic bioreactor operating at a DOC of $50 \%$ of air saturation to the anaerobic bioreactor (DOC $=0 \%$ and $\mathrm{N}_{2}$ sparging). Regarding bioreactors geometry, agitation and circulation conditions, it could be assumed as a first approximation that the two mixing vessels used were similar to homogeneous Continuous Stirred-Tank Reactor (CSTR) vessels while connection tubes could be assumed to real plug-flow bioreactors. Using a constant power number in the turbulent regime for the impeller $\left(N_{p 0}=4\right)$ and the model proposed by Nienow [1], the mixing times in both vessels were also estimated between 1 and $2 \mathrm{~s}$. Thus, these values were two orders of magnitude lower than the mean residence time (Table 1). Lastly, the characteristic time of oxygen consumption from 50 to $0 \%$ of air saturation was previously estimated at $25 \mathrm{~s}$ [27], which is one order of magnitude higher than the mixing time. This confirms the validity of bioreactor homogeneity for the set-up proposed.

Usually, the mean residence time is used in scale-down studies to characterize the set-up and the period of dissolved oxygen (or other substrates) oscillations of concentration . However, mixed bioreactors also perform a mixing of the microbial populations through the residence time distributions (RTD) imposed. While in the case of PF bioreactors, it could be supposed that RTD only consists in a delay function, in the case of CSTR, for a mean residence time $\tau$, the RTD $E(t)$ takes the well-known following form:

$$
E(t)=\frac{1}{\tau} \exp (-t / \tau)
$$


Supposing that the bacteria perfectly followed the liquid flow, the succession of DOC oscillations encountered was thus specific to each bacterium and related to the RTD distributions in each CSTR, which indeed represented the probability for a given entity to spend a time $t$ in the bioreactor. It is thus necessary to first validate the robustness of the set-up by quantifying the dispersion of the aeration ratio $\alpha$ around its mean value given by equation (1). Aiming at this, the oscillations encountered by a statistically representative population of 1,000 microbial sub-populations were modeled using a dedicated Matlab script. From the oscillations profiles obtained, it was then possible to calculate the dynamic fraction of time spent in aerobic conditions $\beta(\mathrm{j}, \mathrm{M})$ for each sub-population $j$ after $M$ circulations or turns inside the set-up. Regarding the residence time in each connection pipe, it was considered that the pipe connecting aerobic compartment to anaerobic one promoted aerobic culture conditions while the other one applied anaerobic environment. Thus, the dynamic ratio $\beta(j, M)$ was determined using the following expression:

$$
\beta(j, M)=\frac{\sum_{i=1}^{M}\left[t_{1}(i, j)\right]+\left(M V_{\text {tube }}\right) / Q}{\sum_{i=1}^{M}\left[t_{1}(i, j)+t_{2}(i, j)\right]+\left(2 M V_{\text {tube }}\right) / Q} j \in 1 \ldots 1,000
$$

With $t_{1}(i, j)$ and $t_{2}(i, j)$ the times spent by each individual bacteria group $j$ in aerobic and anaerobic conditions. The values of $t_{1}(i, j)$ and $t_{2}(i, j)$ were randomly determined in the distribution functions provided by equation 2 and by using the dedicated function of the Statistics and Machine Learning toolbox of Matlab2018 software. The value of $M$ was chosen to match the simulation time to the experimental culture duration. The variation of $\beta$ obtained for $\alpha=0.33$ (experiment 4) and $\alpha=0.67$ (experiment 5) are given in Figure 3A-B. It can be noticed that, as ex- 
pected each dynamic profile of aerobic fractions progressively converged to the mean value $\alpha$ (equation 1 ) but also that some of the bacteria may exhibit sensibly higher or lower values of aeration fraction, especially during the first hours of culture. As a complementary result, the equivalent RTD of the scale-down set-up was determined using closed-loop equivalent model, simulated using the Simulink package of Matlab software. Details of the model the model are given in Supplementary 1. At time $t=0$, a Dirac pulse of numerical tracer was injected at the inlet of compartment 1 while its concentration was recored at the outlet of compartment 2. Each CSTR compartment was modeled using a first-order model in Laplace space while pipes were described by a pure delay model. Volume-averaged tracer concentration $\langle c\rangle$ was also calculated. Simulation results are reported in Figure 4. Simulations 3-5 and 4-6, which only consisted in a permutation of compartments 1 and 2 provided the same RTD distributions as compartment order has no impact on the resulting RTD. These distributions show that the number-averaged value of aeration rate progressively reach the equilibrium value with a time varying from $600 \mathrm{~s}$ to $2000 \mathrm{~s}$ approximately, depending on the operating conditions applied. However, it should be kept in mind that, once this mean value reached, some discrepancies may still exist from one sous-group to another, as indicated by results of Figure 3.

\subsection{Cultures under oscillating DOC conditions}

To study the impact of dissolved oxygen gradients encountered by cells in largescale bioreactors, C. glutamicum $2262 \Delta l d h A$ was continuously circulated between the aerobic and anaerobic compartments of the scale-down set-up. The impact of DOC oscillations on microbial growth kinetics and glucose consumption were de- 
picted in Figure 5. When the cells were cultivated in fully aerobic conditions (reference culture), the maximal biomass concentration reached a value of $19 \mathrm{~g}_{C D W}$ $\mathrm{L}^{-1}$ (Figure 5A). For a mean aeration fraction $\alpha=0.67$, no significant difference was observed between experiments 5 and 6 and the maximal biomass accumulated was $16.3 \mathrm{~g}_{C D W} \mathrm{~L}^{-1}$. Compared to the reference culture, circulation between aerobic and anaerobic environment with this mean aeration fraction resulted in a biomass concentration decrease of approximately $14 \%$. Similar growth were also obtained comparing mean aeration fractions of $\alpha=0.33$ (experiments 3 and 4) but, comparatively to reference culture, a decrease of $31 \%$ in maximal biomass concentration was noticed. Thus, experiments 3 to 6 seemed to indicate that, in the range of culture conditions studied, increasing the residence time while maintaining mean aeration fraction had no sensible influence on bacterial growth. For lowest mean aeration fractions of $\alpha=0.25$ and 0.2 , an important decrease of maximal biomass concentration was observed with a $37 \%$ and $51 \%$ decrease in comparison with reference culture, respectively. Lastly, when $C$. glutamicum $2262 \Delta l d h A$ was cultured in two anaerobic bioreactors $(\alpha=0)$, no sensible growth was observed, resulting in a loss of 96 $\%$ of the maximal biomass concentration compared to the reference culture (Figure $5 \mathrm{~A})$. Moreover, Figure 6 showed that $\mu_{\max }$ increased linearly with the mean aeration fraction $\alpha$ and varied between $0.02 \mathrm{~h}^{-1}$ and $0.6 \mathrm{~h}^{-1}$ for $\alpha=0$ and $\alpha=1$, respectively. This would indicate that the apparent specific growth rate of the bacteria was strongly related to mean fraction of aeration and would thus also impact the time necessary to reach the maximal biomass concentration $t\left(X_{\max }\right)$. Indeed, the report of the quantity $1 / t\left(X_{\max }\right)$ also indicated a linear relationship between both variables 
(Figure 6). It could be thus suggested that the aerobic oxidative metabolism of the bacteria could sequentially operate, without lag phase but with a loss of productivity, as indicated by the decrease of the maximal biomass concentration (Figure 5). This may indicate an apparent and number-averaged metabolism able to rapidly switch from aerobic to anaerobic culture conditions ; this will be further discussed in the paragraph dedicated to kinetics modeling (paragraph 3.5).

Influence of DOC oscillations on the organic acids production by $C$. glutamicum $2262 \Delta l d h A$ was also investigated. No significant concentrations of organic acids were measured for aeration fraction strictly higher than 0 . This could probably be explained by the fact that the rapid transition from aerobic to anaerobic culture conditions do not promote organic acids production, as demonstrated in a preceding paper [26] but also that, faced to low glucose concentrations, C. glutamicum 2262 $\Delta l d h A$ may use organic acids as carbon source for its growth [27]. Concerning glucose consumption and according to Figure 5B, the fastest glucose consumption was obtained for the reference culture $\left(\alpha=B i_{R e f 1}\right.$ and $\left.B i o_{R e f 2}\right)$ with a total glucose exhaustion, which was observed within 10 hours. For the lower mean aeration ratios, glucose was also totally consumed later than for the reference cultures. Indeed, in the absence of aeration $(\alpha=0), 90 \%$ of glucose remained in the culture (Figure 5B). According to substrate flux distribution, when cultivated under one aerobic or two interconnected aerobic bioreactors, the carbon was thus mainly converted into biomass with maximal biomass on glucose yield $Y_{X / S}$ of $0.5 \mathrm{~g}_{C D W} \mathrm{~g}_{\text {glucose }}^{-1}$ (Figure 7). This yield progressively decreased when the mean fraction of aeration also decreased. Thus, in comparison with the fully aerobic reference culture, maximal yield 
showed a $56 \%$ decrease in the scale-down experiment with mean aeration fraction of 0.2. Indeed, this yield dropped to $0.07 \mathrm{~g}_{C D W} \mathrm{~g}_{\text {glucose }}^{-1}$ for the fully anaerobic culture. It could be also noted that maximal biomass concentration and maximal specific glucose consumption rate exhibited similar linear trends while, similarly to maximal specific growth rate, maximal biomass to glucose yield was not linearly linked to the mean fraction of aeration. This would thus further suggest the hypothesis of 'on/off' oxidative metabolism of $C$. glutamicum during their culture in the scale-down set-up.

\subsection{Growth kinetic modeling using a RTD-Monod model}

To get further insight in the microbial physiological response, it was proposed to couple the results of RTD modeling with a Monod growth kinetic model. First, Monod law was applied to model the growth of each simulated bacteria $i$, characterized by their concentration $X_{i}$ :

$$
\frac{d X_{i}}{d t}=\mu_{\max } \frac{[G l u c]}{[G l u c]+K_{s}} X_{i}-k_{d} X_{i}, \quad i \in\{1 \ldots 1,000\}
$$

A cell death rate was added in the growth kinetics to model the decrease of biomass concentration observed experimentally (Figure 5A) and the shape of growth kinetics for each mean fraction of aeration ; death kinetics of group $i$ was thus modeled using a first-order kinetic equation $k_{d} X_{i}$. The total biomass concentration was then directly obtained by summing the concentration of each group $i$ : $X=$

$\sum_{i}^{N} X_{i}$. For glucose consumption, a constant biomass to glucose yield was supposed:

$$
\frac{d[G l u c])}{d t}=-\frac{\mu X}{Y_{X / S}}
$$


The kinetic modeling was thus based on these hypotheses.

1. Only $N=1000$ groups of bacteria were tracked inside the two bioreactors to decrease calculation time. Each group thus represented an initial biomass concentration of $X_{0} / N \mathrm{~g}_{C D W} \mathrm{~L}^{-1}$.

2. Kinetic parameters, namely maximal specific growth rate $\mu_{\max }$, biomass on substrate yield $Y_{X / S}$, death rate $k_{d}$ and half-velocity constant $K_{s}$ were maintained constant whatever the culture modeled. Whereas $\mu_{\max }, Y_{X / S}$ and $k_{d}$ were determined using kinetic data of the fully aerobic reference culture, $K_{s}$ was given by Seletzky [29]. The values of the kinetic parameters were given in Table 2.

3. In the aerobic bioreactor, bacteria grew and consumed glucose, whatever the DOC oscillations imposed before (no history effect) and following equations 4 and5. It was also assumed that transition from anaerobic to aerobic metabolism was immediate.

4. In the anaerobic bioreactor, bacteria neither grew nor produced organic acids nor consumed glucose and only the death rate was applied on biomass concentration. In fact, at every run, the bacteria switched promptly from aerated to non-aerated zones, whereas a suitable induction of anaerobic metabolism requires a progressive transition from aerobic to anaerobic [26]. Growth and glucose consumption were thus supposed immediately stopped when the microorganisms entered the anaerobic bioreactor.

The results obtained by the RTD-kinetic model were given in Figure 8. It can be noted that the results were in very good agreement with the experimental mea- 
surement of biomass concentrations. In particular, the coupled RTD-growth kinetic model was able to satisfactory predict the decrease of maximal biomass concentration $X_{\max }$ and the increase of the time necessary to reach $X_{\max }$ when the mean fraction of aeration was increased. This result confirmed the fact that, if a 'on/off' metabolism was supposed for the oxidative metabolism of each bacterium, the global physiological response of the whole microbial population was matching the experimental observations. As noticed before, the operating mode of culture chosen in the present paper was batch for the culture medium despite the fed-batch mode is now preferred at the industrial scale. The main consequence of this choice was an earlier depletion of glucose and thus of microbial growth. However, as the focus was mainly put on the impact of DOC oscillations during the growth phase, this had no impact on the results of the model. Furthermore the earlier death after glucose depletion was taken into account in the model through the death rate $k_{d}$.

\section{Discussion}

This study provided new insights into growth responses of C. glutamicum 2262 $\triangle l d h A$ when subjected to quantified oscillating DOC. The comparison of reference fully aerobic cultures either performed in a single aerobic compartment or two interconnected aerobic bioreactors revealed unchanged growth and metabolic responses (Figure 2A-D). However, subjecting C. glutamicum $2262 \Delta l d h A$ to various mean aeration fractions $\alpha$ by continuously circulating bacterial cells between an aerobic and an anaerobic bioreactor had various impacts on cell growth and organic acids production. For instance, for experiments 5 and $6(\alpha=0.67)$, DOC oscillations with 
residence times in the anaerobic bioreactor of $138 \mathrm{~s}$ and $551 \mathrm{~s}$ respectively (Table 1 and Figure 3) resulted only in a small decrease (14\%) of maximal biomass concentration and glucose uptake rate despite the bacteria were oxygenated only $67 \%$ of total culture time. Scale-down experiments using stirred tank bioreactor (STR) and plug flow reactor (PFR) for the culture of C. glutamicum ATCC 13032 under DOC oscillations with residence times in anaerobic vessel of 45 and $87 \mathrm{~s}$ with total oxygenation ratio of $82 \%$, have also reported unchanged growth yields [30]. In our case, the time spent in anaerobic bioreactor was sensibly higher, which could explain the decrease observed. It is also interesting to notice that biomass concentration decrease was also observed in scale-down systems, with anaerobic residence times of the same order of magnitude, but for other microorganisms such as Saccharomyces cerevisiae [31]. When the anaerobic residence time was increased to $551 \mathrm{~s}$ while aerobic residence time was $138 \mathrm{~s}$ (condition $\alpha=0.2$ in Table 1) or anaerobic residence of $616 \mathrm{~s}$ associated to aerobic residence time of $206 \mathrm{~s}$ (condition $\alpha=0.25$ in Table 1), detrimental consequences on microbial growth and glucose consumption were observed (Figure 5). In two-compartment scale-down experiment, comparing plug-flow and stirred tank characteristics, Limberg et al. [21] observed a $25 \%$ decrease in growth rate and noted an interesting similarity between that decrease and the two-compartment volume proportion. Like in our study, the reduction was explained by growth stop in the oxygen-deprived compartment. By simulating DOC oscillations effects on B. subtilis, using a scale-down approach, Amanullah et al. [32] reported a similar decrease in maximal biomass concentration and specific growth rate when the circulation time was increased at a constant ratio of volume aerobic 
reactor/volume anaerobic reactor. Decreasing the mean aeration ratio resulted in lengthening the culture time. Similarly to our results, Oosterhuis et al. [33] reported detrimental influence of oscillating DOC, not only on gluconic acid productivity but also on the rate of growth of Gluconobacter oxydans. In our scale-down study, minor quantities of organic acids were obtained when C. glutamicum was subjected to DOC fluctuations. Indeed, in C. glutamicum, it is well known that the lack of oxygen and nitrate as external electron acceptors in such conditions resulted in cell growth stop but still in fermentation of carbohydrates into organic acids $[15,34,35]$. When $C$. glutamicum $2262 \Delta l d h A$ was cultured in two interconnected anaerobic bioreactors $(\alpha=0)$, the concentration of organic acids was maximal although growth was almost undetectable. For the other experiments $(\alpha>0)$, organic acids were produced and re-consumed as a second substrate as already reported in previous work [27] in shaking flasks. Metabolization of produced organic acids have also been reported in STR - PFR scale down of C. glutamicum ; the majority of glucose entering the PFR was indeed transformed into lactate in the PFR and which was further quickly metabolized once the bacteria were in the aerated STR again [30]. Through the different scale-down experiments, our results showed that decreasing the volume ratio resulted in a negative impact on growth performance while fermentation products accumulation were slightly enhanced but still anecdotic. Recent scale-down studies involving different strains of $C$. glutamicum performed in a two compartment STR-PFR system, revealed no loss of process performance with DOC oscillation frequencies in the minute range. Indeed, analysis of proteome, metabolome, transcriptome indicated that no change was noted in the response to DOC oscillations. 
Therefore, they emphasized the robustness of $C$. glutamicum in comparison to other industrial microorganisms $[18,30]$. This conclusion was verified in our study when experiments were performed with mean aeration fractions between 0.67 and 1 . For these conditions, the physiological response of $C$. glutamicum was not significantly different from those of fully-aerobic reference culture (Figure 5). However, when mean aeration fraction were below 0.33, maximal substrate uptake rate decreased from $1.3 \mathrm{~g} \mathrm{~g} \mathrm{~g}_{C D W}^{-1} \mathrm{~h}^{-1}$ to $0.2 \mathrm{~g} \mathrm{~g}_{C D W}^{-1} \mathrm{~h}^{-1}$ (Figure 7) associated to bacterial growth performance loss and overflow of side product increase. However, although C. glutamicum showed robustness, Lemoine et al. [19] showed in two and three scale-down that mimic substrate and oxygen gradients, alterations in extra and intra-cellular metabolite concentrations. For example, lysine product yield was seen to be reduced in scale-down cultivation compared to cultivations at homogeneous conditions. To deepen the understanding on $C$. glutamicum performance in scale down tailoring the industrial conditions, Lemoine et al. [20] applied their three-compartment system with complex medium based on several carbon sources and observed drastic reduction of growth and volumetric product yield of a cadaverine with accumulation of many short chain fatty acids. Finally, C. glutamicum has been demonstrated to be able to switch with $100 \mathrm{~s}$ from aerobic respiration to fermentative pathways $[36,37]$ ; these times were similar to the ones studied in the present work and thus confirm the agreement of our results with literature data. Lastly, our study proposed an original coupled RTD-growth model that considered independently 1000 microbial groups based on their respective residence time in each vessel. A very good agreement was obtained between our experimental data and model simulations results. In 
particular, this suggested that the growth of $C$. glutamicum $2262 \Delta l d h A$ observed in our scale-down set-up could be modeled by considering kinetic parameters (maximal specific growth rate, biomass on glucose yield) that were independent of the mean aeration fraction. Thus, these parameters could be determined in aerobic culture conditions and their use extended to scale-down experiments. To our knowledge, growth kinetics modeling in scale-down systems, considering the RTD of the systems is, to date, original in literature.

\section{Conclusion}

Using a two CSTR scale-down set-up, the impact of mean aeration fraction on C. glutamicum $2262 \Delta l d h A$ growth and organic acids production was experimentally studied and modeled using a RTD-kinetic model. Our results showed that, in the range of culture conditions studied, C. glutamicum $2262 \Delta l d h A$ was strongly related to the value of mean aeration fraction but that the organic acids production was nonsignificant due to microbial reuptake. The model results were in excellent agreement without kinetic parameter fitting. Indeed, it could be shown that fully aerobic kinetic data could be directly transposed to oscillating DOC culture conditions.

\section{Acknowledgments}

The authors would like to thank the french ICEEL Carnot Institute for its financial support. 
Variables

$\begin{array}{lll}\text { DOC } & \text { Dissolved Oxygen Concentration } & (\% \text { air sat. }) \\ k_{d} & \text { Death rate } & \left(\mathrm{s}^{-1}\right) \\ M & \text { Number of circulations } & () \\ N & \text { Agitation rate } & \left(\mathrm{s}^{-1}\right) \\ N p_{0} & \text { Power number } & () \\ Q & \text { Circulation flow rate } & \left(\mathrm{m}^{3} \mathrm{~s}^{-1}\right) \\ P & \text { Power dissipation } & \left(\mathrm{W}^{2}\right) \\ \operatorname{Re} & \text { Reynolds number } & () \\ V_{1} & \text { Volume of aerobic bioreactor } & \left(\mathrm{m}^{3}\right) \\ V_{2} & \text { Volume of anaerobic bioreactor } & \left(\mathrm{m}^{3}\right) \\ V_{t u b e} & \text { Volume connection tube } & \left(\mathrm{m}^{3}\right) \\ \text { vvm } & \text { Volume of gas per volume of liquid and per minute } & \left(\mathrm{L} \mathrm{L} \mathrm{L}^{-1} \mathrm{~h}^{-1}\right) \\ X & \text { Fitted biomass concentration } & \left(\mathrm{g}_{C D W} \mathrm{~L}^{-1}\right) \\ X & \text { Biomass concentration } & \left(\mathrm{g}_{C D W} \mathrm{~L}^{-1}\right)\end{array}$

Greek letters

$\begin{array}{lll}\alpha & \text { Aeration ratio } & () \\ \beta & \text { Dynamic fraction of aeration } & () \\ \eta & \text { Dynamic viscosity } & (\mathrm{Pa} \mathrm{s}) \\ \rho & \text { Density } & \left(\mathrm{kg} \mathrm{m}^{-3}\right) \\ \mu & \text { Specific growth rate } & \left(\mathrm{h}^{-1}\right) \\ \tau_{1} & \text { Mean residence time in compartment } 1 & (\mathrm{~s}) \\ \tau_{2} & \text { Mean residence time } 42 \text { compartment } 2 & (\mathrm{~s})\end{array}$




\section{References}

[1] A. Nienow, On impeller circulation and mixing effectiveness in the turbulent flow regime, Chemical Engineering Science 52 (1997) 2557-2565.

[2] A. R. Lara, E. Galindo, O. T. Ramírez, L. A. Palomares, Living with heterogeneities in bioreactors, Molecular Biotechnology 34 (2006) 355-381.

[3] A. Sweere, K. C. A. Luyben, N. Kossen, Regime analysis and scale-down: tools to investigate the performance of bioreactors, Enzyme and Microbial Technology 9 (1987) 386-398.

[4] P. K. Namdev, N. Irwin, B. Thompson, M. R. Gray, Effect of oxygen fluctuations on recombinant Escherichia coli fermentation, Biotechnology and Bioengineering 41 (1993) 666-670.

[5] F. Bylund, F. Guillard, S.-O. Enfors, C. Trägårdh, G. Larsson, Scale down of recombinant protein production: a comparative study of scaling performance, Bioprocess and Biosystems Engineering 20 (1999) 377-389.

[6] C. J. Hewitt, N.-V. Caron, B. Axelsson, C. M. McFarlane, A. W. Nienow, et al., Studies related to the scale-up of high-cell-density Escherichia coli fedbatch fermentations using multiparameter flow cytometry: Effect of a changing microenvironment with respect to glucose and dissolved oxygen concentration, Biotechnology and Bioengineering 70 (2000) 381-390.

[7] N. Oosterhuis, N. Kossen, Dissolved oxygen concentration profiles in a 
production-scale bioreactor, Biotechnology and Bioengineering 26 (1984) 546550.

[8] E. A. Sandoval-Basurto, G. Gosset, F. Bolívar, O. T. Ramírez, Culture of Escherichia coli under dissolved oxygen gradients simulated in a two-compartment scale-down system: Metabolic response and production of recombinant protein, Biotechnology and Bioengineering 89 (2005) 453-463.

[9] A. R. Lara, L. Leal, N. Flores, G. Gosset, F. Bolívar, O. T. Ramírez, Transcriptional and metabolic response of recombinant Escherichia coli to spatial dissolved oxygen tension gradients simulated in a scale-down system, Biotechnology and Bioengineering 93 (2006) 372-385.

[10] C. Langheinrich, A. W. Nienow, et al., Control of ph in large-scale, free suspension animal cell bioreactors: alkali addition and ph excursions, Biotechnology and Bioengineering 66 (1999) 171-179.

[11] F. Bylund, E. Collet, S.-O. Enfors, G. Larsson, Substrate gradient formation in the large-scale bioreactor lowers cell yield and increases by-product formation, Bioprocess Engineering 18 (1998) 171-180.

[12] J. Bryant, The characterization of mixing in fermenters, Advances in Biochemical Engineering 5 (1977) 101-123.

[13] L. Eggeling, H. Sahm, L-glutamate and l-lysine: traditional products with impetuous developments, Applied Microbiology and Biotechnology 52 (1999) $146-153$. 
[14] T. Hermann, Industrial production of amino acids by coryneform bacteria, Journal of Biotechnology 104 (2003) 155-172.

[15] M. Inui, S. Murakami, S. Okino, H. Kawaguchi, A. A. Vertès, H. Yukawa, Metabolic analysis of Corynebacterium glutamicum during lactate and succinate productions under oxygen deprivation conditions, Journal of Molecular Microbiology and Biotechnology 7 (2004) 182-196.

[16] B. Litsanov, M. Brocker, M. Bott, Toward homosuccinate fermentation: metabolic engineering of Corynebacterium glutamicum for anaerobic production of succinate from glucose and formate, Applied and Environmental Microbiology 78 (2012) 3325-3337.

[17] B. Schilling, W. Pfefferle, B. Bachmann, W. Leuchtenberger, W.-D. Deckwer, A special reactor design for investigations of mixing time effects in a scaled-down industrial l-lysine fed-batch fermentation process, Biotechnology and bioengineering 64 (1999) 599-606.

[18] F. Käß, I. Hariskos, A. Michel, H.-J. Brandt, R. Spann, S. Junne, W. Wiechert, P. Neubauer, M. Oldiges, Assessment of robustness against dissolved oxygen/substrate oscillations for Corynebacterium glutamicum dm1933 in twocompartment bioreactor, Bioprocess and Biosystems Engineering 37 (2014) $1151-1162$.

[19] A. Lemoine, N. Maya Martinez-Iturralde, R. Spann, P. Neubauer, S. Junne, Response of Corynebacterium glutamicum exposed to oscillating cultivation condi- 
tions in a two-and a novel three-compartment scale-down bioreactor, Biotechnology and Bioengineering 112 (2015) 1220-1231.

[20] A. Lemoine, M. H. Limberg, S. Kästner, M. Oldiges, P. Neubauer, S. Junne, Performance loss of Corynebacterium glutamicum cultivations under scale-down conditions using complex media, Engineering in Life Sciences 16 (2016) 620-632.

[21] M. H. Limberg, V. Pooth, W. Wiechert, M. Oldiges, Plug flow versus stirred tank reactor flow characteristics in two-compartment scale-down bioreactor: Setup-specific influence on the metabolic phenotype and bioprocess performance of corynebacterium glutamicum, Engineering in Life Sciences 16 (2016) 610-619.

[22] J. Buchholz, M. Graf, A. Freund, T. Busche, J. Kalinowski, B. Blombach, R. Takors, Co2/hco3- perturbations of simulated large scale gradients in a scale-down device cause fast transcriptional responses in Corynebacterium glutamicum, Applied Microbiology and Biotechnology 98 (2014) 8563-8572.

[23] C. Haringa, W. Tang, A. T. Deshmukh, J. Xia, M. Reuss, J. J. Heijnen, R. F. Mudde, H. J. Noorman, Euler-lagrange computational fluid dynamics for (bio) reactor scale down: an analysis of organism lifelines, Engineering in life sciences 16 (2016) 652-663.

[24] C. Haringa, A. T. Deshmukh, R. F. Mudde, H. J. Noorman, Euler-lagrange analysis towards representative down-scaling of a $22 \mathrm{~m} 3$ aerobic s. cerevisiae fermentation, Chemical Engineering Science 170 (2017) 653-669.

[25] C. Haringa, H. J. Noorman, R. F. Mudde, Lagrangian modeling of 
hydrodynamic-kinetic interactions in (bio) chemical reactors: practical implementation and setup guidelines, Chemical Engineering Science 157 (2017) 159168.

[26] A.-K. Kaboré, E. Olmos, M. Fick, F. Blanchard, E. Guedon, S. Delaunay, Aerobiosis-anaerobiosis transition has a significant impact on organic acid production by Corynebacterium glutamicum, Process Biochemistry 52 (2017) 10-21.

[27] A. K. Kaboré, E. Olmos, F. Blanchard, M. Fick, S. Delaunay, E. Guedon, Impact of gas-liquid mass transfer on organic acids production by Corynebacterium glutamicum in unbaffled shake flasks, Biochemical Engineering Journal 101 (2015) 237-247.

[28] A. Schäfer, A. Tauch, W. Jäger, J. Kalinowski, G. Thierbach, A. Pühler, Small mobilizable multi-purpose cloning vectors derived from the Escherichia coli plasmids pk18 and pk19: selection of defined deletions in the chromosome of Corynebacterium glutamicum, Gene 145 (1994) 69-73.

[29] J. M. Seletzky, U. Noak, J. Fricke, E. Welk, W. Eberhard, C. Knocke, J. Büchs, Scale-up from shake flasks to fermenters in batch and continuous mode with Corynebacterium glutamicum on lactic acid based on oxygen transfer and ph, Biotechnology and Bioengineering 98 (2007) 800-811.

[30] F. Käß, S. Junne, P. Neubauer, W. Wiechert, M. Oldiges, Process inhomogeneity leads to rapid side product turnover in cultivation of Corynebacterium glutamicum, Microbial Cell Factories 13 (2014b) 6. 
[31] S. George, G. Larsson, S.-O. Enfors, A scale-down two-compartment reactor with controlled substrate oscillations: metabolic response of Saccharomyces cerevisiae, Bioprocess and Biosystems Engineering 9 (1993) 249-257.

[32] A. Amanullah, B. C. Buckland, A. W. Nienow, Mixing in the fermentation and cell culture industries, Wiley Online Library, 2004.

[33] N. Oosterhuis, N. Kossen, A. Olivier, E. Schenk, Scale-down and optimization studies of the gluconic acid fermentation by Gluconobacter oxydans, Biotechnology and Bioengineering 27 (1985) 711-720.

[34] H. B. T. Khuat, A. K. Kabore, E. Olmos, M. Fick, J. Boudrant, J.-L. Goergen, S. Delaunay, E. Guedon, Lactate production as representative of the fermentation potential of Corynebacterium glutamicum 2262 in a one-step process, Bioscience, Biotechnology and Biochemistry 78 (2014) 343-349.

[35] H. Dominguez, C. Nezondet, N. Lindley, M. Cocaign, Modified carbon flux during oxygen limited growth of Corynebacterium glutamicum and the consequences for amino acid overproduction, Biotechnology Letters 15 (1993) 449-454.

[36] S. Okino, M. Inui, H. Yukawa, Production of organic acids by Corynebacterium glutamicum under oxygen deprivation, Applied Microbiology and Biotechnology 68 (2005) 475-480.

[37] S. Yamamoto, M. Sakai, M. Inui, H. Yukawa, Diversity of metabolic shift in response to oxygen deprivation in Corynebacterium glutamicum and its close relatives, Applied Microbiology and Biotechnology 90 (2011) 1051-1061. 
${ }_{547}$ Table legends

${ }_{548}$ Table 1. Scale-down culture conditions.

${ }_{549}$ Table 2. Values of the optimized parameters for kinetic modeling. 
Tables

\section{Table 1}

Table 1: Scale-down culture conditions.

\begin{tabular}{|c|c|c|c|c|c|c|c|c|}
\hline \multirow[b]{2}{*}{ Experiment } & \multirow[b]{2}{*}{$\alpha$} & \multirow[b]{2}{*}{$\begin{array}{c}Q \\
(\mathrm{~mL} / \mathrm{s})\end{array}$} & \multicolumn{3}{|c|}{ Compartment 1} & \multicolumn{3}{|c|}{ Compartment 2} \\
\hline & & & $\begin{array}{l}V_{1} \\
(\mathrm{~L})\end{array}$ & $\begin{array}{l}\tau_{1} \\
(\mathrm{~s})\end{array}$ & $\begin{array}{c}\mathrm{DOC}^{1} \\
(\% \text { air sat.)}\end{array}$ & $\begin{array}{l}V_{2} \\
(\mathrm{~L})\end{array}$ & $\begin{array}{l}\tau_{2} \\
(\mathrm{~s})\end{array}$ & $\begin{array}{c}\mathrm{DOC}^{2} \\
(\% \text { air sat) }\end{array}$ \\
\hline reference $\mathrm{Bio}_{r e f 1}$ & $1^{(4)}$ & $\mathrm{n} / \mathrm{a}$ & 1.5 & $\mathrm{n} / \mathrm{a}$ & 50 & $\mathrm{n} / \mathrm{a}$ & $\mathrm{n} / \mathrm{a}$ & $\mathrm{n} / \mathrm{a}$ \\
\hline reference $\mathrm{Bio}_{r e f 2}$ & $1^{(3)}$ & 7.3 & 1.5 & 205 & 50 & 1.5 & 205 & 50 \\
\hline reference $\mathrm{Bio}_{r e f 3}$ & 0 & 7.3 & 1.5 & 205 & $0^{(1)}$ & 1.5 & 205 & 0 \\
\hline 1 & 0.2 & 7.3 & 1 & 137 & 50 & 4 & 548 & 0 \\
\hline 2 & 0.25 & 7.3 & 1.5 & 205 & 50 & 4.5 & 616 & 0 \\
\hline 3 & 0.33 & 7.3 & 1 & 137 & 50 & 2 & 274 & 0 \\
\hline 4 & 0.33 & 1.8 & 1 & 556 & 50 & 2 & 1111 & 0 \\
\hline 5 & 0.67 & 7.3 & 2 & 274 & 50 & 1 & 137 & 0 \\
\hline 6 & 0.67 & 1.8 & 2 & 1111 & 50 & 1 & 556 & 0 \\
\hline
\end{tabular}

(1) In this case, once the oxygen probe was calibrated, the culture was started without air/oxygen sparging nor nitrogen sparging whereas the $\mathrm{Bio}_{N 2}$ compartment was sparged continuously with nitrogen.

(2) Nitrogen was sparged continuously to warrant anaerobic conditions.

(3) Corresponded to two aerobic bioreactors interconnected.

(4) Corresponded to a single anaerobic bioreactor. 


\section{Table 2}

Table 2: Values of the optimized parameters for kinetic modeling

\begin{tabular}{lllll}
\hline Parameter & $\mu_{\max }\left(\mathrm{h}^{-1}\right)$ & $Y_{X / S}\left(\mathrm{~g} \mathrm{~g}_{C D W}^{-1}\right)$ & $K_{s}\left(\mathrm{~g} \mathrm{~L}^{-1}\right)$ & $k_{d}\left(\mathrm{~h}^{-1}\right)$ \\
\hline \hline Value & $0.55^{*}$ & $0.4^{*}$ & $0.0045^{* *}$ & $0.0005^{*}$ \\
\hline
\end{tabular}

$(*)$ Parameters determined using the fully aerobic reference culture $\operatorname{Bio}_{R e f 1}$. $(* *)$ Value given by [29]. 


\section{Figure legends}

Figure 1. Experimental set-up.

Figure 2. Reference culture kinetics of C. glutamicum $2262 \Delta l d h A$ under constant dissolved oxygen concentration. Temporal profiles of biomass concentration and glucose concentrations in a single bioreactor with constant dissolved oxygen concentration of $50 \%$ of air saturation (A) and in two interconnected stirred tank bioreactors with constant dissolved oxygen concentration of $50 \%$ of air saturation (B). Temporal profiles of biomass concentration and glucose concentration (C) and organic acids concentration (D) in two interconnected stirred anaerobic bioreactors. The different symbols represent: glucose $(\square)$, biomass $(\bullet)$, succinate $(*)$, acetate $(\triangleleft)$, pyruvate $(\diamond)$.

Figure 3. Variation of the dynamic fraction of aeration $\beta$ for 10,000 bacteria in function of time for mean aeration fractions $\alpha=0.33(\mathrm{~A})$ and $\alpha=0.67$ (B).

Figure 4. Modeled Residence Time Distribution in the scale-down set-up normalized by volume-averaged tracer concentration $\langle c\rangle$. Operating conditions as given by Table 1 were experiment $1,(\cdot \cdot)$, experiment $2(\cdot-\cdot-\cdot)$, experiment 3 and $5\left(--_{-}\right)$, experiments 4 and $6(-)$.

Figure 5. Influence of dissolved oxygen oscillation on C. glutamicum $2262 \Delta l d h A$ growth and substrate consumption during a scale-down cultures. (A). Growth kinet- 
579

582

583

ics; (B). Glucose uptake profiles. The mean aerobic fractions $\alpha$ are: $0(\times), 0.2(*)$, $0.25(\bullet), 0.33$ - experiment $3(\diamond), 0.33$ - experiment $4(\diamond), 0.67$ - experiment $5(\mathbf{\square})$, 0.67 - experiment $(\square) 0.67,1 \mathrm{a}(\bullet), 1 \mathrm{~b}(\circ)$.

Figure 6. Relationship between mean fraction of aeration $\alpha, 1 / t\left(X_{\max }\right)(\square)$ and maximal specific growth rate $\mu_{\max }(\Delta)$.

Figure 7. Relationship between mean fraction of aeration $\alpha$, maximal biomass concentration $X_{\max }(\square)$, maximal specific glucose consumption rate (o) and maximal biomass on glucose yield $Y_{X / S}(\Delta)$.

Figure 8. Comparison of experimental and modeled growth kinetics and impact of DOC oscillations. Dots are experimental values while continuous lines are modeled kinetics. Mean aerobic fractions $\alpha$ are $0.2(\square,-\bullet \bullet-), 0.25(\diamond,---), 0.33-$ experiment $3(\Delta,-\bullet), 0.67$ - experiment $5(*, \bullet \bullet \bullet), 1(+,-)$. 
Figure 1

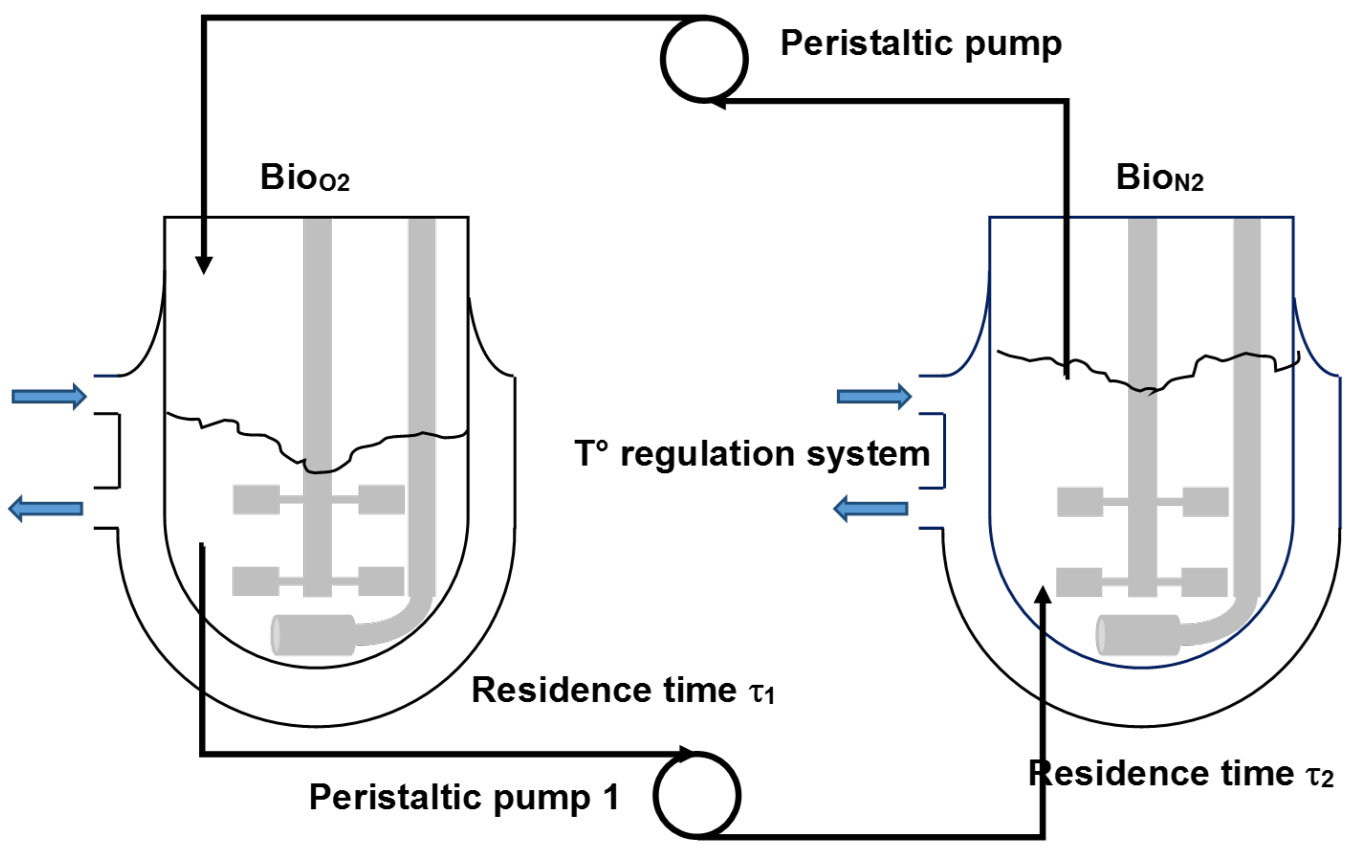

Figure 1: Experimental set-up 


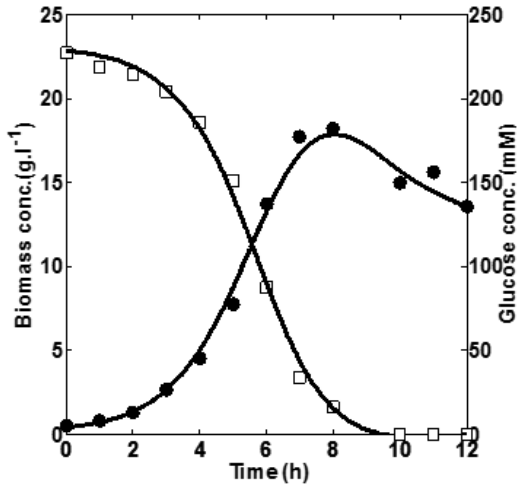

(A)

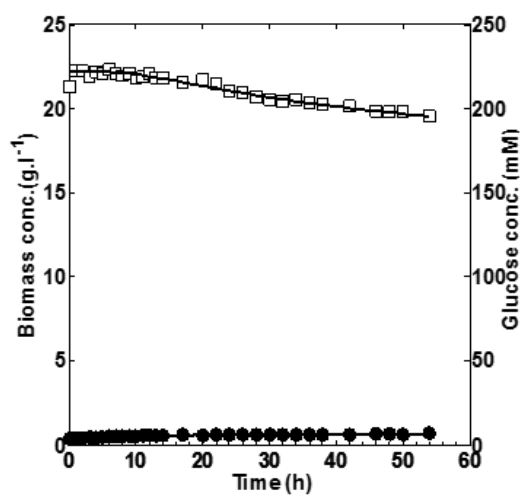

(C)

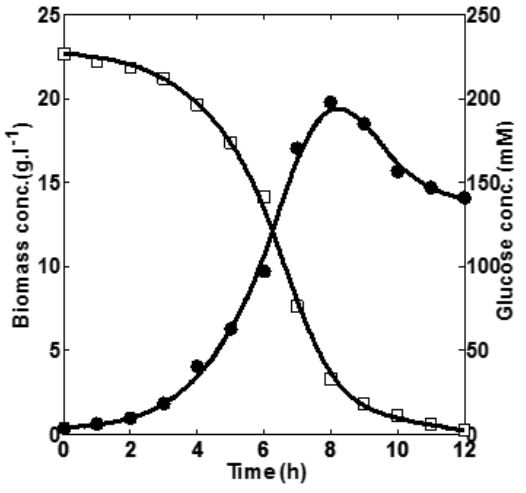

(B)

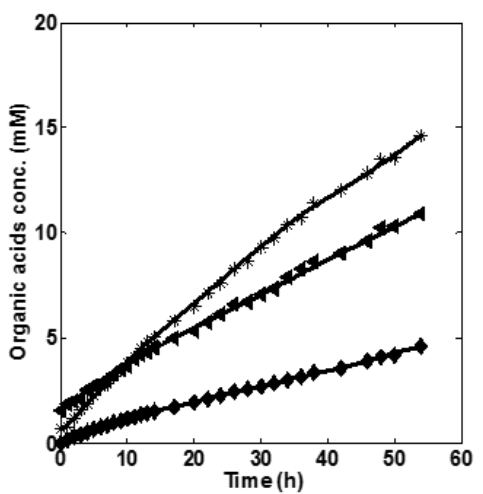

(D)

Figure 2: Reference culture kinetics of C. glutamicum $2262 \Delta l d h A$ under constant dissolved oxygen concentration. Temporal profiles of biomass concentration and glucose concentrations in a single bioreactor with constant dissolved oxygen concentration of $50 \%$ of air saturation (A) and in two interconnected stirred tank bioreactors with constant dissolved oxygen concentration of $50 \%$ of air saturation (B). Temporal profiles of biomass concentration and glucose concentration (C) and organic acids concentration (D) in two interconnected stirred anaerobic bioreactors. The different symbols represent: glucose $(\square)$, biomass $(\bullet)$, succinate $(*)$, acetate $(\triangleleft)$, pyruvate $(\bullet)$. 
Figure 3

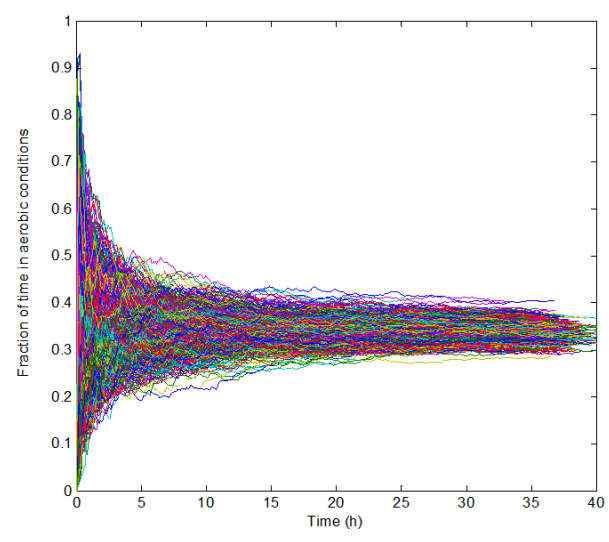

(A)

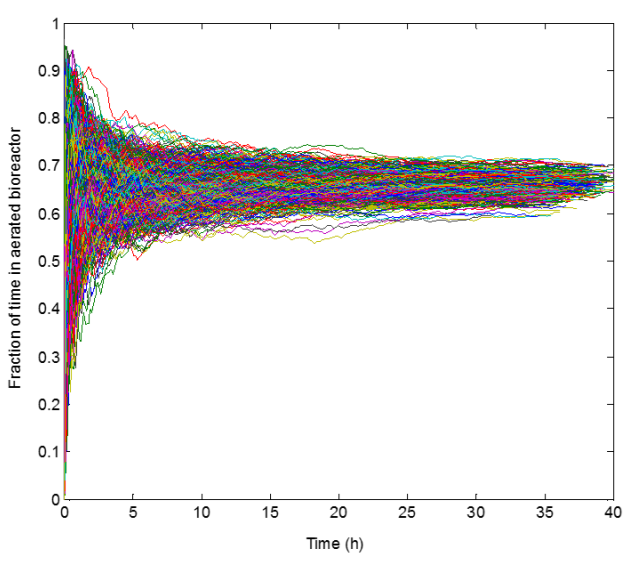

(B)

Figure 3: Variation of the dynamic fraction of aeration $\beta$ for 10,000 bacteria in function of time for mean aeration fractions $\alpha=0.33(\mathrm{~A})$ and $\alpha=0.67(\mathrm{~B})$. 


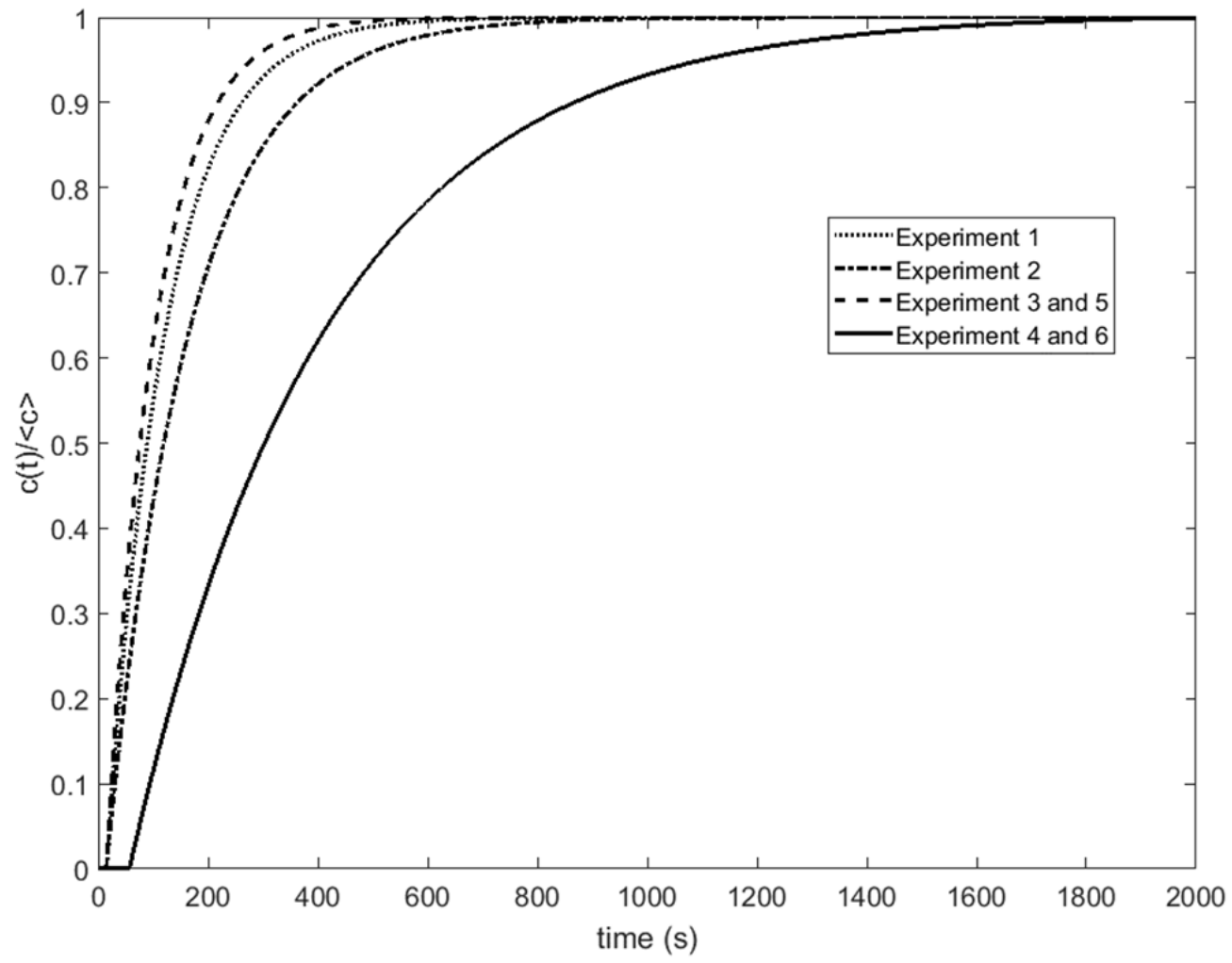

Figure 4: Modeled Residence Time Distribution in the scale-down set-up normalized by volumeaveraged tracer concentration $\langle c\rangle$. Operating conditions as given by Table 1 were experiment 1 , $(\cdots)$, experiment $2(\cdot-\cdot-\cdot)$, experiment 3 and $5(--)$, experiments 4 and $6(-)$. 

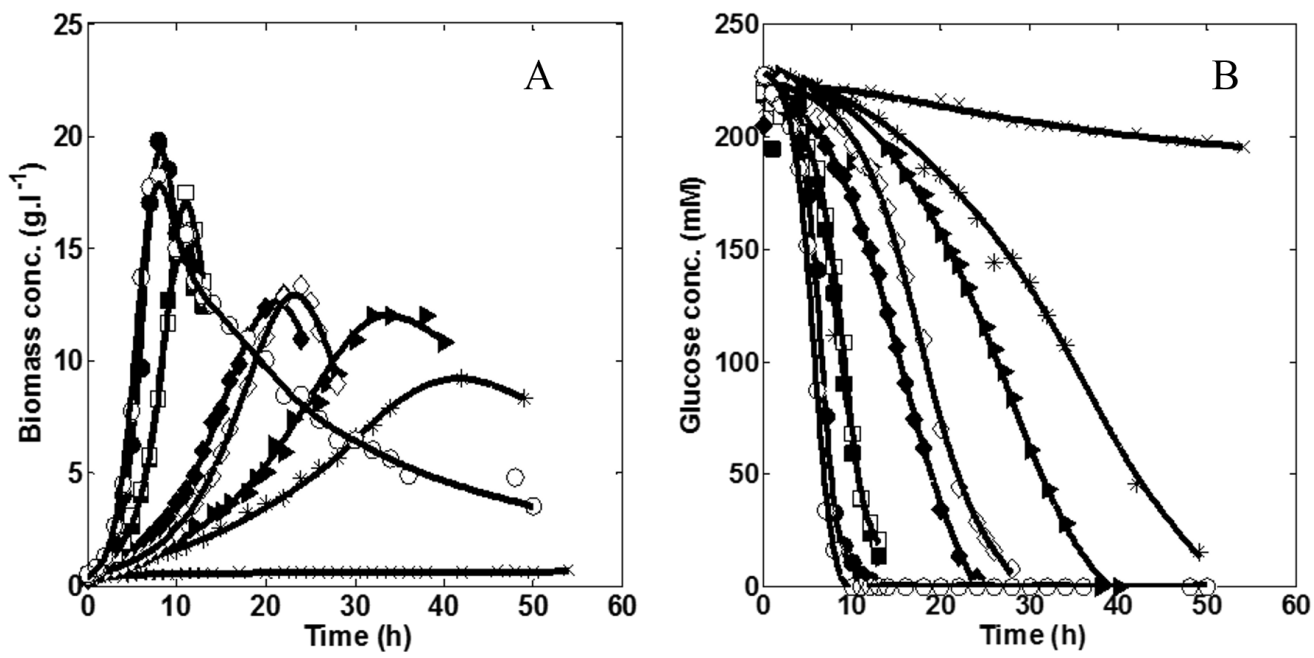

Figure 5: Influence of dissolved oxygen oscillation on C. glutamicum $2262 \Delta l d h A$ growth and substrate consumption during a scale-down cultures. (A). Growth kinetics; (B). Glucose uptake profiles. The mean aerobic fractions $\alpha$ are: $0(\times), 0.2(*), 0.25(\bullet), 0.33-$ experiment $3(\diamond), 0.33$ experiment $4(\diamond), 0.67$ - experiment $5(\mathbb{\square}), 0.67$ - experiment $(\square) 0.67,1 \mathrm{a}(\bullet), 1 \mathrm{~b}(\circ)$. 


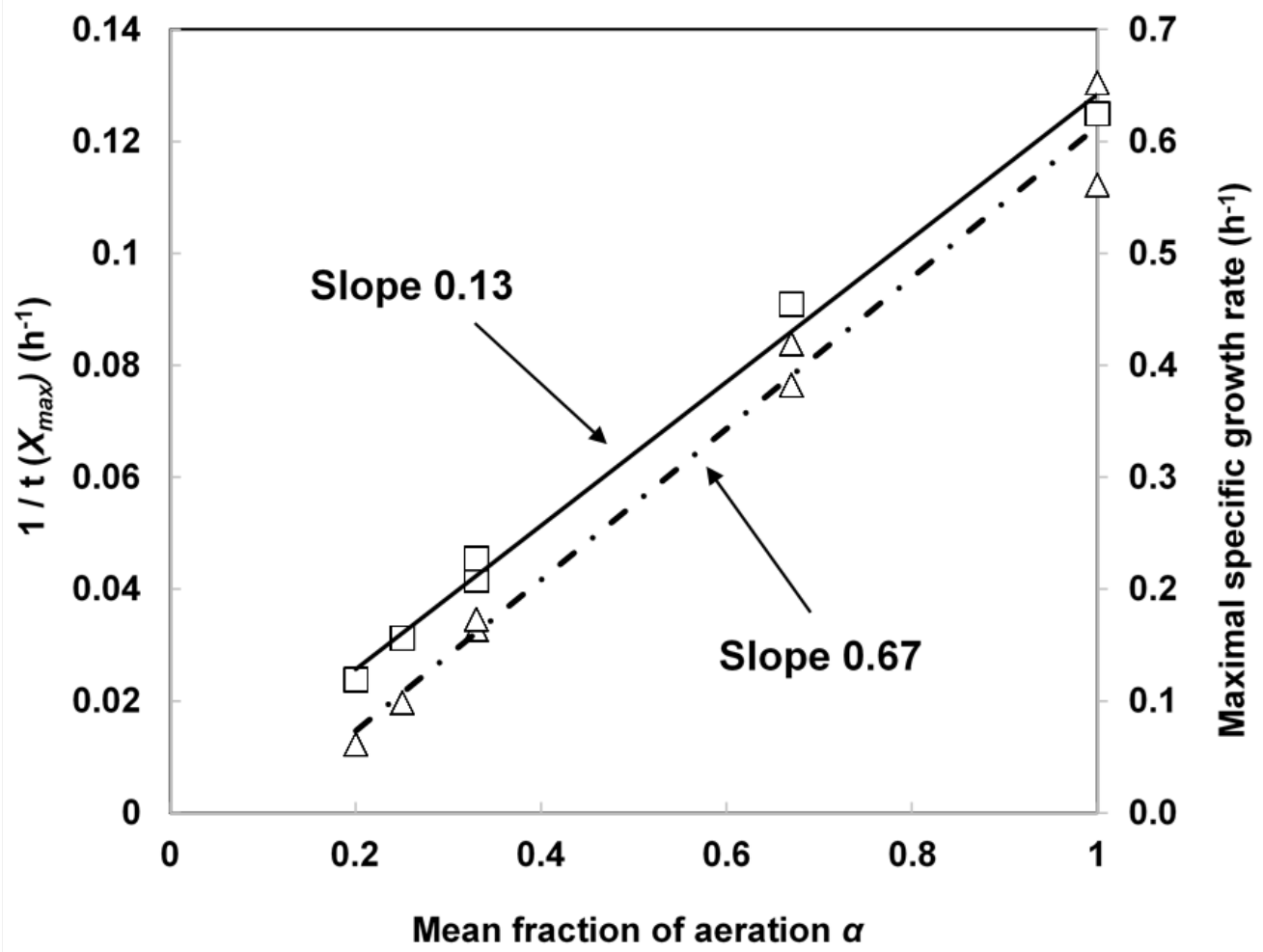

Figure 6: Relationship between mean fraction of aeration $\alpha, 1 / t\left(X_{\max }\right)(\square)$ and maximal specific growth rate $\mu_{\max }(\Delta)$. 


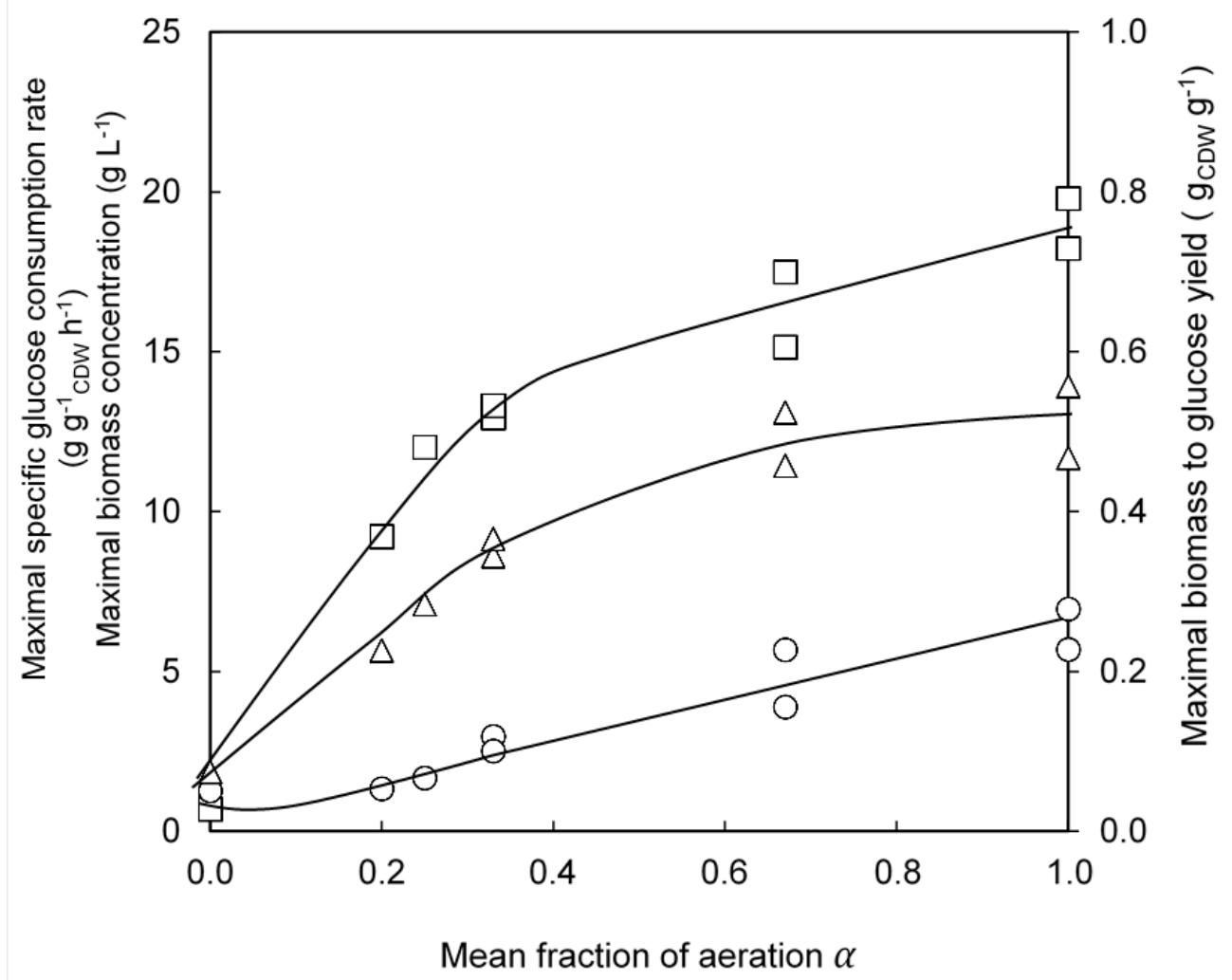

Figure 7: Relationship between mean fraction of aeration $\alpha$, maximal biomass concentration $X_{\max }$ $(\square)$, maximal specific glucose consumption rate (o) and maximal biomass on glucose yield $Y_{X / S}$ $(\Delta)$. 


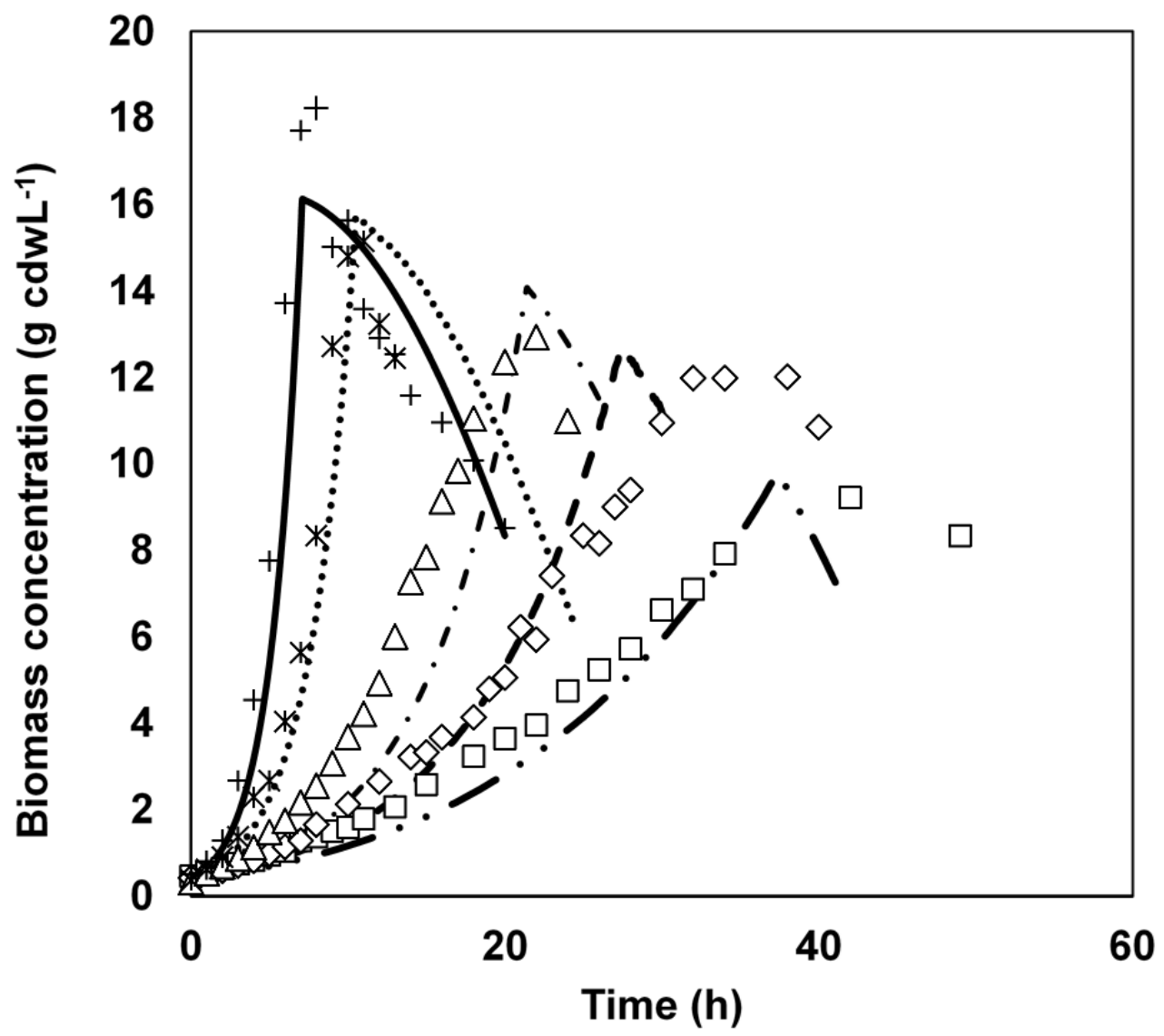

Figure 8: Comparison of experimental and modeled growth kinetics and impact of DOC oscillations. Dots are experimental values while continuous lines are modeled kinetics. Mean aerobic fractions $\alpha$ are $0.2(\square,-\bullet \bullet), 0.25(\diamond,---), 0.33$ - experiment $3(\Delta,-\bullet-), 0.67$ - experiment $5(*, \bullet \bullet \bullet), 1(+$, $-)$. 


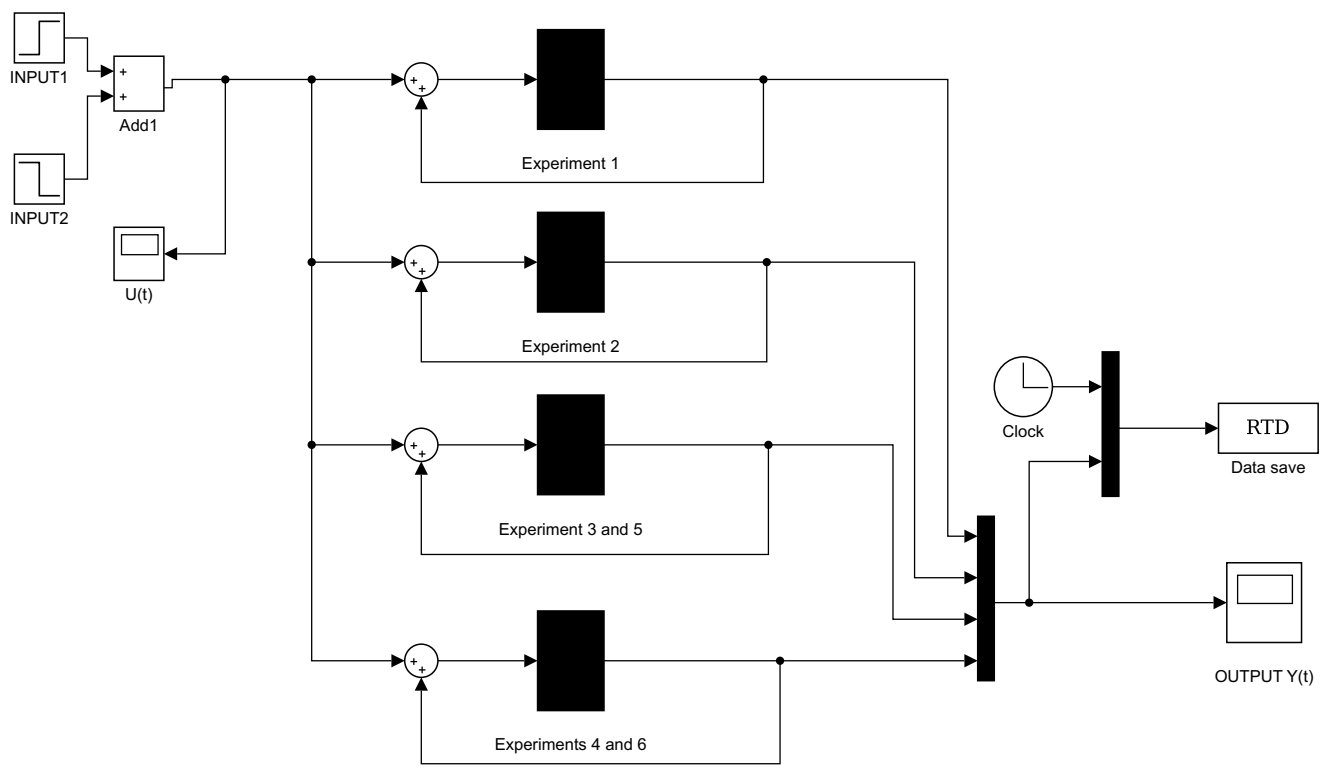

The Simulink model defined for each culture condition was reported on Figure 9.

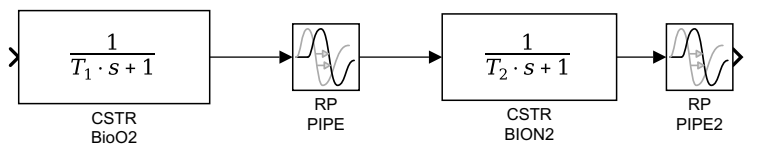

Figure 9: Modeling of scale-down set-up using Simulink software. 

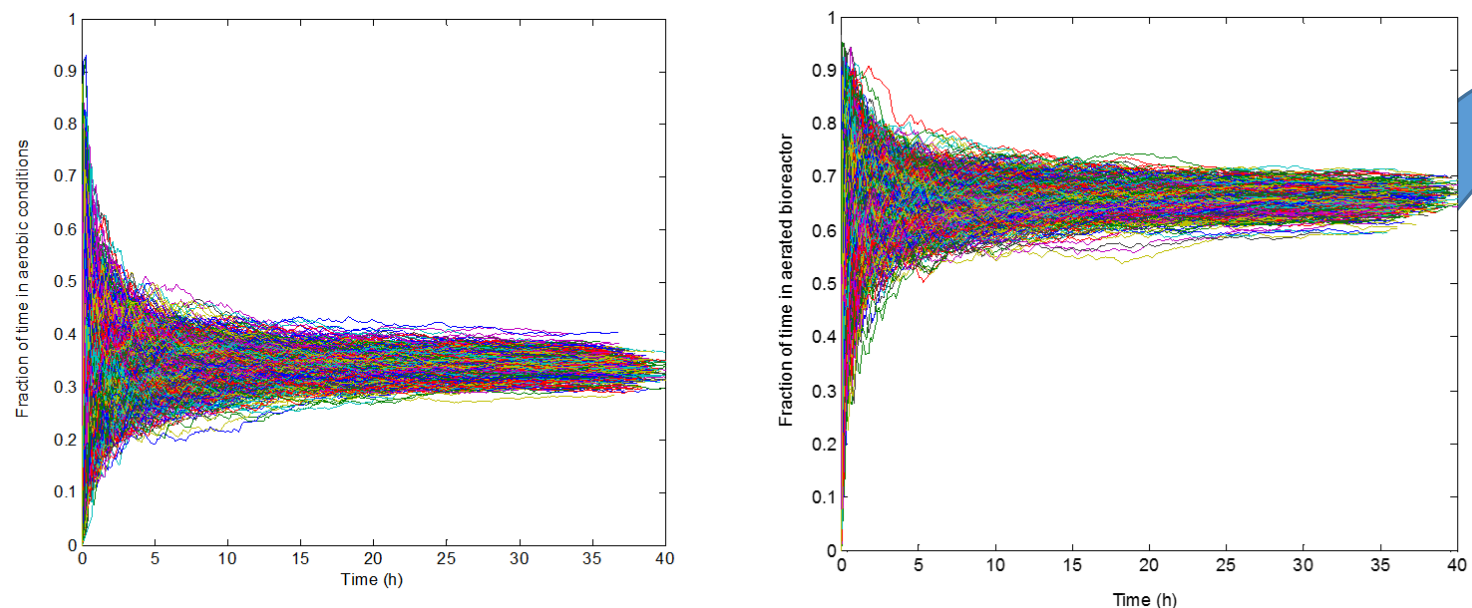

Modelling of lagrangian tracking of the bacteria

$$
+
$$

Experimental study of $C$. glutamicum growth in a twocompartment scale-down bioreactor, imposing fluctuating $\mathrm{dO}_{2}$.
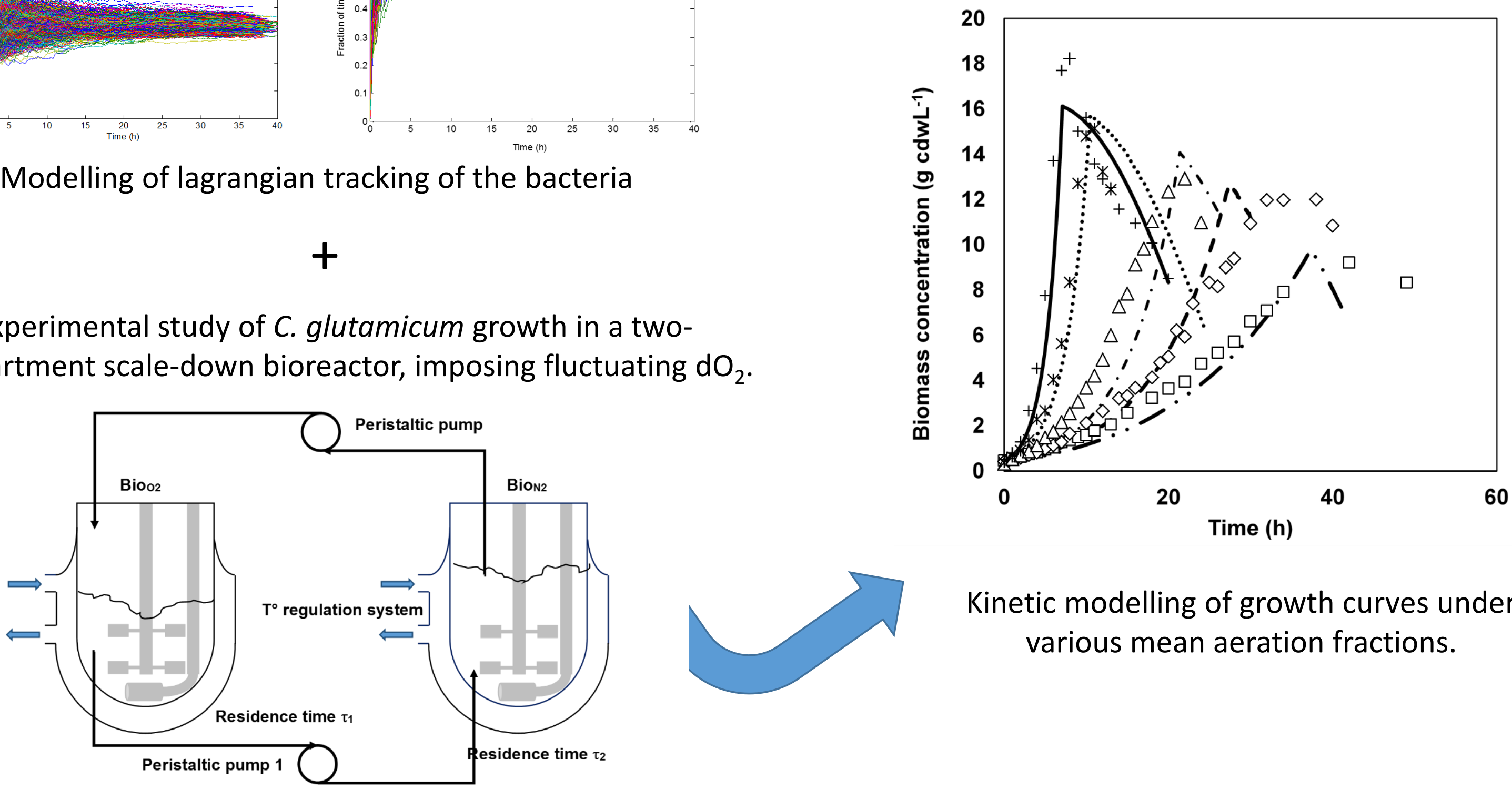

Kinetic modelling of growth curves under various mean aeration fractions. 